\title{
On the interpolation of the measure of non-compactness of bilinear operators with weak assumptions on the boundedness of the operator
}

\author{
Fernando Cobos * \\ Departamento de Análisis Matemático y Matemática Aplicada, Facultad de Matemáticas, \\ Universidad Complutense de Madrid, Plaza de Ciencias 3, 28040 Madrid. Spain. \\ Luz M. Fernández-Cabrera \\ Sección Departamental del Departamento de Análisis Matemático y Matemática Aplicada, \\ Facultad de Estudios Estadísticos, Universidad Complutense de Madrid, 28040 Madrid, \\ Spain. \\ Antón Martínez \\ Departamento de Matemática Aplicada I, Escuela de Ingeniería Industrial, Universidad de \\ Vigo, 36200 Vigo, Spain.
}

\begin{abstract}
We complete the range of the parameters in the interpolation formula established by Mastyło and Silva for the measure of non-compactness of a bilinear operator interpolated by the real method.
\end{abstract}

Keywords: Bilinear operators; measure of non-compactness; duality for bilinear operators; real interpolation.

2010 MSC: Primary 46B70, 46M35, 47H60.

Dedicated to Professor Hans Triebel on the occasion of his 85th birthday.

\section{Introduction}

One of the questions considered by Calderón [4] in his seminal paper on the complex method was the interpolation of compact bilinear operators. The counterpart for the real method of Lions and Peetre [18] has been done recently, starting with the papers by Fernandez and Silva [11] and Fernández-Cabrera and Martínez [12, 13]. A motivation for the research has been the fact that compact bilinear operators arise rather naturally in harmonic analysis. Namely, commutators of bilinear Calderón-Zygmund operators and multiplication by functions in the subspace CMO of BMO are compact (see the papers by Bényi and Torres [1], Cobos, Fernández-Cabrera and Martínez [6] and Torres, Xue and Yan $[26])$. Other results on interpolation of compact bilinear operators can be

\footnotetext{
* Corresponding author.

Email addresses: cobos@mat.ucm.es (Fernando Cobos ), luz_fernandez-c@mat.ucm.es (Luz M. Fernández-Cabrera), antonmar@uvigo.es (Antón Martínez )
} 
found in the papers by Mastyło and Silva [22] and Cobos, Fernández-Cabrera and Martínez [7, 8].

Quantitative versions in terms of the measure of non-compactness of some of these qualitative results have been established by Mastyło and Silva [21] and Besoy and Cobos [3]. Both papers refer to the real method but they work with different assumptions on the operator. Mastyło and Silva assume that $T$ is defined from $\left(A_{0} \cap A_{1}\right) \times\left(B_{0} \cap B_{1}\right)$ into $E_{0} \cap E_{1}$ with

$$
\|T(a, b)\|_{E_{j}} \leq M_{j}\|a\|_{A_{j}}\|b\|_{B_{j}}, a \in A_{0} \cap A_{1}, b \in B_{0} \cap B_{1}, j=0,1,
$$

and establish the following log-convexity inequality for the measure of noncompactness

$$
\begin{aligned}
& \beta\left(T: \bar{A}_{\theta, p} \times \bar{B}_{\theta, q} \longrightarrow \bar{E}_{\theta, r}\right) \\
& \quad \leq C \beta\left(T: A_{0}^{\circ} \times B_{0}^{\circ} \longrightarrow E_{0}^{\circ}\right)^{1-\theta} \beta\left(T: A_{1}^{\circ} \times B_{1}^{\circ} \longrightarrow E_{1}^{\circ}\right)^{\theta}
\end{aligned}
$$

where $1 \leq p, q<\infty, 1<r<\infty$ and $1 / p+1 / q=1+1 / r$ (see [21, Theorem 3.2]). Besoy and Cobos ask a stronger assumption on $T$. Namely, they suppose that $T$ is bounded from $\left(A_{0}+A_{1}\right) \times\left(B_{0}+B_{1}\right)$ into $E_{0}+E_{1}$ and that the restrictions $T: A_{j} \times B_{j} \longrightarrow E_{j}$ are also bounded for $j=0,1$. In this case, they show that the log-convexity inequality holds for operators acting among couples of quasi-Banach spaces and for the whole range of the parameters $p, q, r$ (see $[3$, Theorem 3.5]). More precisely, if $\left(E_{0}, E_{1}\right)$ is a couple of $s$-Banach spaces, then the conditions on the parameters are

$$
0<p, q, r \leq \infty \text { with } 1 / r= \begin{cases}1 / p+1 / q-1 / s & \text { if } \quad p, q \geq s \\ 1 / \max (p, q) & \text { if } \quad p<s \text { or } q<s .\end{cases}
$$

Approaches followed in [21] and [3] are completely different.

In applications, the weaker assumption on $T$ is more handy. For this reason, it is important to extend inequality (1.1) to the remaining range of parameters. For couples of Banach spaces, this means the values of (1.2) with $s=1$. Accordingly, we prove in this paper such a result.

Our techniques are a refinement of those used by Mastyło and Silva [21] based on duality results for bilinear operators introduced by Ramanujan and Schock [24] and the corresponding results established by Cobos, FernándezMartínez and Martínez [9] and Fernández-Martínez [14] for the measure of noncompactness in the linear case (see also the paper by Edmunds and Teixeira [25]). Duality is the reason why we work with Banach couples.

We start by reviewing in Section 2 the basic results on the real interpolation method. We also establish there the variant of the bilinear interpolation theorem that we will need later. In Section 3 we study the connections between the measure of non-compactness of a bilinear operator and the measure of noncompactness of its adjoint operator, which is a linear operator. Finally, in Section 4 , we establish the log-convexity inequality.

\section{Preliminaries}

By a Banach couple $\bar{A}=\left(A_{0}, A_{1}\right)$ we mean two Banach spaces $A_{0}, A_{1}$ which are continuously embedded in some Hausdorff topological vector space. 
We endow $A_{0}+A_{1}=\Sigma(\bar{A})$ and $A_{0} \cap A_{1}=\Delta(\bar{A})$ with the norms $K(1, \cdot)$ and $J(1, \cdot)$, respectively, where for $t>0$

$$
K(t, a)=\inf \left\{\left\|a_{0}\right\|_{A_{0}}+t\left\|a_{1}\right\|_{A_{1}}: a=a_{0}+a_{1}, a_{j} \in A_{j}\right\}, a \in \Sigma(\bar{A}),
$$

and

$$
J(t, a)=\max \left\{\|a\|_{A_{0}}, t\|a\|_{A_{1}}\right\}, a \in \Delta(\bar{A}),
$$

are the Peetre's functionals.

Let $0<\theta<1$ and $0<q \leq \infty$. The real interpolation space $\left(A_{0}, A_{1}\right)_{\theta, q}=$ $\bar{A}_{\theta, q}$ is formed by all $a \in A_{0}+A_{1}$ such that

$$
\|a\|_{\bar{A}_{\theta, q}}=\left(\int_{0}^{\infty}\left[t^{-\theta} K(t, a)\right]^{q} \frac{d t}{t}\right)^{1 / q}<\infty,
$$

(the integral should be replaced by the supremum when $q=\infty$ ). The space $\left(A_{0}, A_{1}\right)_{\theta, q}$ is a Banach space if $1 \leq q \leq \infty$ and a quasi-Banach space if $0<q<1$ (see $[2,27])$.

The real interpolation method has the interpolation property for linear operators: Let $\bar{B}=\left(B_{0}, B_{1}\right)$ be another Banach couple and let $R$ be a linear operator from $A_{0}+A_{1}$ into $B_{0}+B_{1}$ whose restrictions $R: A_{j} \longrightarrow B_{j}$ are bounded for $j=0,1$. Then $R: \bar{A}_{\theta, q} \longrightarrow \bar{B}_{\theta, q}$ is bounded with

$$
\|R\|_{\bar{A}_{\theta, q}, \bar{B}_{\theta, q}} \leq\|R\|_{A_{0}, B_{0}}^{1-\theta}\|R\|_{A_{1}, B_{1}}^{\theta} .
$$

The space $\left(A_{0}, A_{1}\right)_{\theta, q}$ can also be introduced using series instead of integrals because the functional

$$
\|a\|_{\theta, q}=\left(\sum_{m=-\infty}^{\infty}\left[2^{-\theta m} K\left(2^{m}, a\right)\right]^{q}\right)^{1 / q}
$$

(the sum should be replaced by the supremum if $q=\infty$ ) is an equivalent norm (respectively, quasi-norm) to $\|\cdot\|_{\bar{A}_{\theta, q}}$ if $1 \leq q \leq \infty$ (respectively, if $0<q<1$ ).

The real interpolation space can be equivalently described by means of the $J$-functional. Indeed, $\left(A_{0}, A_{1}\right)_{\theta, q}$ consists of all $a \in A_{0}+A_{1}$ for which there is $\left(u_{m}\right) \subseteq A_{0} \cap A_{1}$ such that

$$
a=\sum_{m=-\infty}^{\infty} u_{m} \quad\left(\text { convergence in } A_{0}+A_{1}\right)
$$

and

$$
\left(\sum_{m=-\infty}^{\infty}\left[2^{-\theta m} J\left(2^{m}, u_{m}\right)\right]^{q}\right)^{1 / q}<\infty
$$

Moreover, the functional

$\|a\|_{\theta, q}^{J}=\inf \left\{\left(\sum_{m=-\infty}^{\infty}\left[2^{-\theta m} J\left(2^{m}, u_{m}\right)\right]^{q}\right)^{1 / q}: a=\sum_{m=-\infty}^{\infty} u_{m},\left(u_{m}\right) \subseteq A_{0} \cap A_{1}\right\}$

is equivalent to $\|\cdot\|_{\bar{A}_{\theta, q}}$.

Subsequently, if $A$ is a quasi-Banach space, we put $A^{*}$ for its dual space. If $A_{0} \cap A_{1} \subseteq A$, we write $A^{\circ}$ for the closure of $A_{0} \cap A_{1}$ in $A$. Since $A_{0} \cap A_{1}=$ 
$A_{0}^{\circ} \cap A_{1}^{\circ}$, it follows from the $J$-description of the real interpolation space that $\left(A_{0}, A_{1}\right)_{\theta, q}=\left(A_{0}^{\circ}, A_{1}^{\circ}\right)_{\theta, q}$. The $J$-description also gives that $A_{0} \cap A_{1}$ is dense in $\bar{A}_{\theta, q}$ if $q<\infty$. Therefore, $\bar{A}_{\theta, q}^{\circ}=\bar{A}_{\theta, q}$ if $0<q<\infty$.

We say that the Banach couple $\bar{A}$ is regular if $A_{0} \cap A_{1}$ is dense in $A_{0}$ and in $A_{1}$. If this is the case, then $\overline{A^{*}}=\left(A_{0}^{*}, A_{1}^{*}\right)$ is also a Banach couple and the following duality formulae hold with equivalence of norms:

(2.1) If $1 \leq q<\infty, 1 / q+1 / q^{\prime}=1$ and $0<\theta<1$, then

$$
\left(A_{0}, A_{1}\right)_{\theta, q}^{*}=\left(A_{0}^{*}, A_{1}^{*}\right)_{\theta, q^{\prime}} .
$$

(2.2) If $q=\infty$ and $0<\theta<1$, then

$$
\left(\left(A_{0}, A_{1}\right)_{\theta, \infty}^{\circ}\right)^{*}=\left(A_{0}^{*}, A_{1}^{*}\right)_{\theta, 1} .
$$

(2.3) If $0<q<1$ and $0<\theta<1$, then

$$
\left(A_{0}, A_{1}\right)_{\theta, q}^{*}=\left(A_{0}^{*}, A_{1}^{*}\right)_{\theta, \infty} .
$$

See [2, Theorem 3.7.1 and Remark in p. 55] and [23, (0.5) in p. 124].

Let $A, B, E$ be Banach spaces and let $T: A \times B \longrightarrow E$ be a bilinear operator. We say that $T$ is bounded if

$$
\|T\|_{A \times B, E}=\sup \left\{\|T(a, b)\|_{E}:\|a\|_{A} \leq 1,\|b\|_{B} \leq 1\right\}<\infty .
$$

We write $\mathcal{L}(A \times B, E)$ for the space of all bounded bilinear operators from $A \times B$ into $E$.

Let $\bar{E}=\left(E_{0}, E_{1}\right)$ be another Banach couple. By $T \in \mathcal{B}(\bar{A} \times \bar{B}, \bar{E})$ we mean that $T$ is a bilinear operator defined on $\left(A_{0} \cap A_{1}\right) \times\left(B_{0} \cap B_{1}\right)$ with values in $E_{0} \cap E_{1}$ such that there are constants $M_{j}>0$ with

$$
\|T(a, b)\|_{E_{j}} \leq M_{j}\|a\|_{A_{j}}\|b\|_{B_{j}}, a \in \Delta(\bar{A}), b \in \Delta(\bar{B}), j=0,1 .
$$

Using (2.4), it is not hard to check that $T$ may be uniquely extended to a bilinear operator $T: A_{j}^{\circ} \times B_{j}^{\circ} \longrightarrow E_{j}^{\circ}$ with $\|T\|_{A_{j}^{\circ} \times B_{j}^{\circ}, E_{j}^{\circ}} \leq M_{j}$.

The behaviour of bounded linear operators under real interpolation was already considered by Lions and Peetre [18] in their foundational paper on the real method. Their result was extended by Karadzhov [16] to the full range for the parameters (see also the paper by König [17] for a proof). In our later considerations we are going to need the following variant of the bilinear interpolation theorem.

Theorem 2.1. Let $\bar{A}=\left(A_{0}, A_{1}\right), \bar{B}=\left(B_{0}, B_{1}\right), \bar{E}=\left(E_{0}, E_{1}\right)$ be Banach couples and let $T$ be a bilinear operator from $\left(A_{0} \cap A_{1}\right) \times\left(B_{0}+B_{1}\right)$ into $E_{0}+E_{1}$ such that there are positive constants $M_{j}$ with

$$
\|T(a, b)\|_{E_{j}} \leq M_{j}\|a\|_{A_{j}}\|b\|_{B_{j}}, a \in A_{0} \cap A_{1}, b \in B_{j}, j=0,1 .
$$

Let $0<\theta<1$ and $0<p, q, r \leq \infty$ be positive numbers satisfying

$$
\frac{1}{r}= \begin{cases}\frac{1}{p}+\frac{1}{q}-1 & \text { if } \quad p, q \geq 1 \\ \frac{1}{\max (p, q)} & \text { if } \quad p<1 \text { or } q<1\end{cases}
$$


Then there is a constant $C>0$ independent of $T$ such that

$$
\|T(a, b)\|_{\bar{E}_{\theta, r}} \leq C M_{0}^{1-\theta} M_{1}^{\theta}\|a\|_{\bar{A}_{\theta, p}}\|b\|_{\bar{B}_{\theta, q}}, \quad a \in \Delta(\bar{A}), b \in \bar{B}_{\theta, q} .
$$

Moreover, $T$ may be uniquely extended to a bounded bilinear operator

$$
T: \bar{A}_{\theta, p}^{\circ} \times \bar{B}_{\theta, q} \longrightarrow \bar{E}_{\theta, r} .
$$

Proof. Take $n \in \mathbb{Z}$ such that $2^{n} \leq M_{1} / M_{0}<2^{n+1}$. Given any $a \in A_{0} \cap A_{1}$, $u \in B_{0} \cap B_{1}$ and $m, k \in \mathbb{Z}$, if $a=a_{0}+a_{1}$ with $a_{j} \in A_{j}$, we obtain

$$
\begin{aligned}
K\left(2^{m}, T(a, u)\right) & \leq\left\|T\left(a_{0}, u\right)\right\|_{E_{0}}+2^{m}\left\|T\left(a_{1}, u\right)\right\|_{E_{1}} \\
& \leq M_{0}\left\|a_{0}\right\|_{A_{0}}\|u\|_{B_{0}}+2^{m} M_{1}\left\|a_{1}\right\|_{A_{1}}\|u\|_{B_{1}} \\
& \leq \max \left(M_{0}, 2^{-n} M_{1}\right)\left(\left\|a_{0}\right\|_{A_{0}}+2^{m-k}\left\|a_{1}\right\|_{A_{1}}\right) J\left(2^{k+n}, u\right) .
\end{aligned}
$$

Hence,

$$
K\left(2^{m}, T(a, u)\right) \leq 2 M_{0} K\left(2^{m-k}, a\right) J\left(2^{k+n}, u\right)
$$

Now take any $b \in \bar{B}_{\theta, q}$ and let $b=\sum_{k=-\infty}^{\infty} u_{k}$ be any $J$-representation of $b$. Since $\sum_{k=-\infty}^{\infty}\left\|u_{k}\right\|_{B_{0}+B_{1}}<\infty$, we also have that $b=\sum_{k=-\infty}^{\infty} u_{k+n}$ in $B_{0}+B_{1}$.

Suppose that $p<1$ and $p \leq q$, so $r=q$. We have

$$
K\left(2^{m}, T(a, b)\right) \leq \sum_{k=-\infty}^{\infty} K\left(2^{m}, T\left(a, u_{k+n}\right)\right) \leq\left(\sum_{k=-\infty}^{\infty} K\left(2^{m}, T\left(a, u_{k+n}\right)\right)^{p}\right)^{1 / p} .
$$

Combining this inequality with (2.5) and using Young's inequality with parameters $p / q=1+p / q-1$, we derive

$$
\begin{aligned}
\|T(a, b)\|_{\bar{E}_{\theta, q}} & \leq 2 M_{0}\left\|\left(\sum_{k=-\infty}^{\infty} 2^{-\theta(m-k) p} K\left(2^{m-k}, a\right)^{p} 2^{-\theta k p} J\left(2^{k+n}, u_{k+n}\right)^{p}\right)\right\|_{\ell_{q / p}}^{1 / p} \\
& \leq 2 M_{0}\left\|\left(2^{-\theta m} K\left(2^{m}, a\right)\right)^{p}\right\|_{\ell_{1}}^{1 / p}\left\|\left(2^{-\theta k} J\left(2^{k+n}, u_{k+n}\right)\right)\right\|_{\ell_{q}} \\
& \leq 2 M_{0} 2^{\theta n}\|a\|_{\theta, p}\left\|\left(2^{-\theta k} J\left(2^{k}, u_{k}\right)\right)\right\|_{\ell_{q}} .
\end{aligned}
$$

Taking the infimum over all $J$-representations of $b$, we derive that

$$
\|T(a, b)\|_{\bar{E}_{\theta, q}} \leq C M_{0}^{1-\theta} M_{1}^{\theta}\|a\|_{\bar{A}_{\theta, p}}\|b\|_{\bar{B}_{\theta, q}}, \quad a \in \Delta(\bar{A}), b \in \bar{B}_{\theta, q} .
$$

Finally, since $A_{0} \cap A_{1}$ is dense in $\bar{A}_{\theta, \underline{p}}$, the operator $T$ may be uniquely extended to a bounded bilinear operator $T: \bar{A}_{\theta, p} \times \bar{B}_{\theta, q} \longrightarrow \bar{E}_{\theta, q}$.

If $q<p \leq 1$, then $r=p$ and we can proceed as before but using now that

$$
K\left(2^{m}, T(a, b)\right) \leq\left(\sum_{k=-\infty}^{\infty} K\left(2^{m}, T\left(a, u_{k+n}\right)\right)^{q}\right)^{1 / q}
$$

and Young's inequality with parameters $q / p=q / p+1-1$. The cases $q<1$ and $q \leq p$, and $p<q \leq 1$ are similar. Finally, if $1 \leq p, q$ then $1 / r=1 / p+1 / q-1$ and we can proceed directly with Young's inequality.

Others results on interpolation of bilinear operators can be found, for example, in the papers by Janson [15], Mastyło [19, 20] and Cobos, Fernández-Cabrera and Martínez [6] . 


\section{Measure of non-compactness}

Let $A, B$ be Banach spaces. We write $U_{A}$ for the closed unit ball of $A$ and define $U_{B}$ similarly. Given any bounded linear operator $R \in \mathcal{L}(A, B)$, the (ball) measure of non-compactness $\beta(R)=\beta(R: A \longrightarrow B)$ is defined to be the infimum of the set of all $\sigma>0$ for which there is a finite subset $\left\{b_{1}, \cdots, b_{s}\right\} \subseteq B$ such that

$$
R\left(U_{A}\right) \subseteq \bigcup_{k=1}^{s}\left\{b_{k}+\sigma U_{B}\right\}
$$

(see $[5,10])$.

Clearly, the operator $R$ is compact if and only if $\beta(R)=0$.

Let $R^{*} \in \mathcal{L}\left(B^{*}, A^{*}\right)$ be the adjoint operator of $R$. A well-known result of Schauder says that $R$ is compact if and only if $R^{*}$ is compact. If the operator $R$ is not compact, then the following inequalities hold for the measure of noncompactness

$$
\frac{1}{2} \beta(R: A \longrightarrow B) \leq \beta\left(R^{*}: B^{*} \longrightarrow A^{*}\right) \leq 2 \beta(R: A \longrightarrow B),
$$

(see [10, Corollary 2.10, p. 12]).

Let $E$ be another Banach spaces and let $T: A \times B \longrightarrow E$ be a bounded bilinear operator. The (ball) measure of non-compactness $\beta(T)=\beta(T: A \times$ $B \longrightarrow E$ ) of $T$ is the infimum of all $\sigma>0$ for which there exists a finite subset $\left\{z_{1}, \cdots, z_{s}\right\} \subseteq E$ such that

$$
T\left(U_{A}, U_{B}\right)=\left\{T(a, b): a \in U_{A}, b \in U_{B}\right\} \subseteq \bigcup_{k=1}^{s}\left\{z_{k}+\sigma U_{E}\right\} .
$$

The operator $T$ is said to be compact if for any bounded sets $V \subseteq A, W \subseteq B$ we have that the closure of $T(V, W)$ is compact in $E$. Again, the operator $T$ is compact if and only if its measure of non-compactness is 0 .

Following Ramanujan and Schock [24], the adjoint operator $T^{\times}$of $T$ is the linear map

$$
T^{\times}: E^{*} \longrightarrow \mathcal{L}(A \times B, \mathbb{K})
$$

defined by $\left(T^{\times} f\right)(a, b)=f[T(a, b)]$. Here $\mathbb{K}$ is the scalar field.

It turns out that $\|T\|_{A \times B, E}=\left\|T^{\times}\right\|_{E^{*}, \mathcal{L}(A \times B, \mathbb{K})}$. Moreover, $T$ is compact if and only if $T^{\times}$is compact (see [24, Theorem 2.6]).

Next we study inequalities of the type (3.1) in the bilinear setting. For operators acting among Banach couples, this question has been studied in [21, Lemma 3.1],

Theorem 3.1. Let $A, B, E$ be Banach spaces and let $T \in \mathcal{L}(A \times B, E)$. Then we have

$$
\beta\left(T^{\times}: E^{*} \longrightarrow \mathcal{L}(A \times B, \mathbb{K})\right) \leq 4 \beta(T: A \times B \longrightarrow E) .
$$

Proof. Given any $\varepsilon>0$, we can find finite subsets $\left\{a_{1}, \cdots, a_{s}\right\} \subseteq U_{A}$ and $\left\{b_{1}, \cdots, b_{s}\right\} \subseteq U_{B}$ such that for any $(a, b) \in U_{A} \times U_{B}$ there exits $1 \leq \bar{k} \leq s$ with

$$
\left\|T(a, b)-T\left(a_{k}, b_{k}\right)\right\| \leq 2 \beta(T)+\varepsilon .
$$

Let $S: E^{*} \longrightarrow \mathbb{K}^{s}$ be the linear operator assigning to each $f \in E^{*}$ the $s$-tuple $S f=\left(f\left[T\left(a_{1}, b_{1}\right)\right], \cdots, f\left[T\left(a_{s}, b_{s}\right)\right]\right)$. Since $S$ has finite rank, $S$ is compact. 
Therefore, there exits a finite subset of functionals $\left\{f_{1}, \cdots, f_{m}\right\} \subseteq U_{E^{*}}$ such that for any $f \in U_{E^{*}}$ there is $1 \leq n \leq m$ with $\left\|S f-S f_{n}\right\|_{\mathbb{K}^{s}} \leq \varepsilon$. Whence,

$$
\left|f\left[T\left(a_{k}, b_{k}\right)\right]-f_{n}\left[T\left(a_{k}, b_{k}\right)\right]\right| \leq \varepsilon \text { for any } 1 \leq k \leq s .
$$

Then, given any $f \in U_{E^{*}}$ if we take $n$ satisfying (3.3), and for any $(a, b) \in$ $U_{A} \times U_{B}$ we choose $k$ satisfying (3.2), we obtain

$$
\begin{aligned}
\mid\left(T^{\times} f-\right. & \left.T^{\times} f_{n}\right)(a, b)|=| f[T(a, b)]-f_{n}[T(a, b)] \mid \\
\leq \mid f[T(a, b)]- & f\left[T\left(a_{k}, b_{k}\right)\right]|+| f\left[T\left(a_{k}, b_{k}\right)\right]-f_{n}\left[T\left(a_{k}, b_{k}\right)\right] \mid \\
& +\left|f_{n}\left[T\left(a_{k}, b_{k}\right)\right]-f_{n}[T(a, b)]\right| \\
\leq & \left\|T(a, b)-T\left(a_{k}, b_{k}\right)\right\|_{E}+\varepsilon+\left\|T\left(a_{k}, b_{k}\right)-T(a, b)\right\|_{E} \leq 4 \beta(T)+3 \varepsilon .
\end{aligned}
$$

This yields that $\left\|T^{\times} f-T^{\times} f_{n}\right\|_{A \times B, \mathbb{K}} \leq 4 \beta(T)+3 \varepsilon$. Consequently,

$$
\beta\left(T^{\times}: E^{*} \longrightarrow \mathcal{L}(A \times B, \mathbb{K})\right) \leq 4 \beta(T: A \times B \longrightarrow E) .
$$

Theorem 3.2. Let $A, B, E$ be Banach spaces and let $T \in \mathcal{L}(A \times B, E)$. Then we have

$$
\beta(T: A \times B \longrightarrow E) \leq 8 \beta\left(T^{\times}: E^{*} \longrightarrow \mathcal{L}(A \times B, \mathbb{K})\right) .
$$

Proof. Put $W=\overline{T^{\times}\left(E^{*}\right)}$ which is a Banach space with the induced norm from $\mathcal{L}(A \times B, \mathbb{K})$. Let $\left\{R_{1}, \ldots, R_{s}\right\} \subseteq \mathcal{L}(A \times B, \mathbb{K})$ and $\sigma>0$ satisfy that

$$
T^{\times}\left(U_{E^{*}}\right) \subseteq \bigcup_{k=1}^{s}\left\{R_{k}+\sigma U_{\mathcal{L}(A \times B, \mathbb{K})}\right\} .
$$

We may assume that $W \cap\left\{R_{k}+\sigma U_{\mathcal{L}(A \times B, \mathbb{K})}\right\} \neq \varnothing$ for $k=1, \ldots, s$. Pick $S_{k} \in W \cap\left\{R_{k}+\sigma U_{\mathcal{L}(A \times B, \mathbb{K})}\right\}$. Then

$$
T^{\times}\left(U_{E^{*}}\right) \subseteq \bigcup_{k=1}^{s}\left\{S_{k}+2 \sigma U_{W}\right\} .
$$

This yields that

$$
\beta\left(T^{\times}: E^{*} \longrightarrow W\right) \leq 2 \beta\left(T^{\times}: E^{*} \longrightarrow \mathcal{L}(A \times B, \mathbb{K})\right) .
$$

Now consider the adjoint operator $\left(T^{\times}\right)^{*}: W^{*} \longrightarrow E^{* *}$ and let $R: A \times B \longrightarrow$ $W^{*}$ the operator defined by $R(a, b)=g_{(a, b)}$ where

$$
g_{(a, b)}\left(T^{\times} f\right)=f[T(a, b)] .
$$

The operator $R$ is bilinear and has norm less than or equal to 1 because

$$
\begin{aligned}
\|R(a, b)\|_{W^{*}} & =\sup \left\{\left|g_{(a, b)}\left(T^{\times} f\right)\right|:\left\|T^{\times} f\right\|_{A \times B, \mathbb{K}} \leq 1\right\} \\
& =\sup \left\{|f[T(a, b)]|:\left\|T^{\times} f\right\|_{A \times B, \mathbb{K}} \leq 1\right\} \\
& =\sup \left\{\left|\left(T^{\times} f\right)(a, b)\right|:\left\|T^{\times} f\right\|_{A \times B, \mathbb{K}} \leq 1\right\} \leq\|a\|_{A}\|b\|_{B} .
\end{aligned}
$$


The following diagram is useful

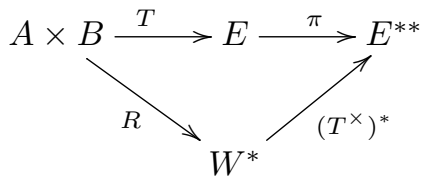

where $\pi(w)=\widehat{w}$ is the natural embedding from $E$ into its bidual $E^{* *}$. Note that the diagram commutes because

$$
\begin{aligned}
\left(\left(T^{\times}\right)^{*} R(a, b)\right)(f) & =R(a, b)\left(T^{\times} f\right)=g_{(a, b)}\left(T^{\times} f\right) \\
& =f[T(a, b)]=\widehat{T(a, b)}(f)=\pi(T(a, b))(f) .
\end{aligned}
$$

Consequently, using the diagram and (3.1), we obtain

$$
\begin{aligned}
\beta(T: A \times B \longrightarrow E) & \leq 2 \beta\left(\pi T: A \times B \longrightarrow E^{* *}\right) \\
& \leq 2\|R\|_{A \times B, W^{*}} \beta\left(\left(T^{\times}\right)^{*}: W^{*} \longrightarrow E^{* *}\right) \\
& \leq 4 \beta\left(T^{\times}: E^{*} \longrightarrow W\right) \\
& \leq 8 \beta\left(T^{\times}: E^{*} \longrightarrow \mathcal{L}(A \times B, \mathbb{K})\right) .
\end{aligned}
$$

\section{Interpolation of the measure of non-compactness}

We start with an auxiliary result which complements an embedding of $[7, \mathrm{p}$. 5] (see also [21, Theorem 2.1]).

Subsequently, for $0<r \leq \infty$ we put

$$
\widetilde{r}=\left\{\begin{array}{lll}
\infty & \text { if } \quad 0<r \leq 1, \\
r^{\prime}, 1 / r+1 / r^{\prime}=1 & \text { if } \quad 1<r \leq \infty .
\end{array}\right.
$$

Lemma 4.1. Let $\bar{A}=\left(A_{0}, A_{1}\right), \bar{B}=\left(B_{0}, B_{1}\right)$ be regular Banach couples. For $j=0,1$, put $X_{j}=\mathcal{L}\left(A_{j} \times B_{j}, \mathbb{K}\right)$ and let $0<\theta<1$ and $0<p, q, r \leq \infty$ such that

$$
\frac{1}{r}= \begin{cases}\frac{1}{p}+\frac{1}{q}-1 & \text { if } \quad p, q \geq 1, \\ \frac{1}{\max (p, q)} & \text { if } \quad p<1 \text { or } q<1 .\end{cases}
$$

Then the following continuous embedding holds

$$
\left(X_{0}, X_{1}\right)_{\theta, \widetilde{r}} \hookrightarrow \mathcal{L}\left(\bar{A}_{\theta, p}^{\circ} \times \bar{B}_{\theta, q}^{\circ}, \mathbb{K}\right) .
$$

Proof. The pair $\bar{X}=\left(X_{0}, X_{1}\right)$ is a Banach couple because $X_{j} \hookrightarrow \mathcal{L}(\Delta(\bar{A}) \times \Delta(\bar{B}), \mathbb{K}), j=0,1$. Let

$$
\Phi: \Delta(\bar{A}) \times \mathcal{L}(\Delta(\bar{A}) \times \Delta(\bar{B}), \mathbb{K}) \longrightarrow \Delta(\bar{B})^{*}
$$

be the bilinear operator defined by $\Phi(a, R)(b)=R(a, b)$. Since

$$
|R(a, b)| \leq\|R\|_{\Delta(\bar{A}) \times \Delta(\bar{B}), \mathbb{K}}\|a\|_{\Delta(\bar{A})}\|b\|_{\Delta(\bar{B})},
$$


the operator $\Phi$ has norm less than or equal to 1 . Moreover, if $a \in \Delta(\bar{A})$ and $R \in X_{j}$, we get

$$
|R(a, b)| \leq\|R\|_{X_{j}}\|a\|_{A_{j}}\|b\|_{B_{j}} .
$$

So

$$
\Phi:\left(\Delta(\bar{A}),\|\cdot\|_{A_{j}}\right) \times X_{j} \longrightarrow B_{j}^{*}, \quad j=0,1,
$$

is also bounded, with norm less than or equal to 1 .

By the duality formulae (2.1), (2.2), (2.3), we know that

$$
\left(\left(B_{0}, B_{1}\right)_{\theta, q}^{\circ}\right)^{*}=\left(B_{0}^{*}, B_{1}^{*}\right)_{\theta, \widetilde{q}}=\bar{B}_{\theta, \widetilde{q}} .
$$

Moreover, we have

$$
\frac{1}{\widetilde{q}}=\left\{\begin{array}{lll}
\frac{1}{p}+\frac{1}{\widetilde{r}}-1 & \text { if } & p \geq 1 \\
\frac{1}{\widetilde{r}} & \text { if } & p<1 .
\end{array}\right.
$$

Whence, applying Theorem 2.1, we get that $\Phi$ may be uniquely extended to a bounded bilinear operator

$$
\Phi: \bar{A}_{\theta, p}^{\circ} \times \bar{X}_{\theta, \widetilde{r}} \longrightarrow{\overline{B^{*}}}_{\theta, \widetilde{q}}=\left(\left(B_{0}, B_{1}\right)_{\theta, q}^{\circ}\right)^{*} .
$$

Consequently, there is a constant $C>0$ such that for any $R \in \bar{X}_{\theta, \widetilde{r}}$ and any $a \in \Delta(\bar{A}), b \in \Delta(\bar{B})$, we have

$$
|R(a, b)|=|\Phi(a, R)(b)| \leq\|\Phi(a, R)\|_{\bar{B}^{*}{ }_{\theta, \widetilde{q}}}\|b\|_{\bar{B}_{\theta, q}} \leq C\|R\|_{\bar{X}_{\theta, \tilde{r}}}\|a\|_{\bar{A}_{\theta, p}}\|b\|_{\bar{B}_{\theta, q}} .
$$

This yields that $R \in \mathcal{L}\left(\bar{A}_{\theta, p}^{\circ} \times \bar{B}_{\theta, q}^{\circ}, \mathbb{K}\right)$ and that the embedding

$$
\bar{X}_{\theta, \widetilde{r}} \hookrightarrow \mathcal{L}\left(\bar{A}_{\theta, p}^{\circ} \times \bar{B}_{\theta, q}^{\circ}, \mathbb{K}\right)
$$

is bounded with norm less than or equal to $C$.

Now we are ready to establish the announced result on interpolation of the measure of non-compactness of bilinear operators.

Theorem 4.2. Let $\bar{A}=\left(A_{0}, A_{1}\right), \bar{B}=\left(B_{0}, B_{1}\right), \bar{E}=\left(E_{0}, E_{1}\right)$ be Banach couples and let $T \in \mathcal{B}(\bar{A} \times \bar{B}, \bar{E})$. Let $0<\theta<1$ and $0<p, q, r \leq \infty$ such that

$$
\frac{1}{r}= \begin{cases}\frac{1}{p}+\frac{1}{q}-1 & \text { if } \quad p, q \geq 1 \\ \frac{1}{\max (p, q)} & \text { if } \quad p<1 \text { or } q<1 .\end{cases}
$$

Then

$$
\begin{aligned}
\beta\left(T: \bar{A}_{\theta, p}^{\circ} \times \bar{B}_{\theta, q}^{\circ}\right. & \left.\longrightarrow \bar{E}_{\theta, r}^{\circ}\right) \\
& \leq C \beta\left(T: A_{0}^{\circ} \times B_{0}^{\circ} \longrightarrow E_{0}^{\circ}\right)^{1-\theta} \beta\left(T: A_{1}^{\circ} \times B_{1}^{\circ} \longrightarrow E_{1}^{\circ}\right)^{\theta},
\end{aligned}
$$

Here $C$ is a constant independent of $T$.

Proof. According to the bilinear interpolation theorem (see, for example [13, Theorem 4.1]), we know that $T$ may be uniquely extend to a bounded bilinear operator $T: \bar{A}_{\theta, p}^{\circ} \times \bar{B}_{\theta, q}^{\circ} \longrightarrow \bar{E}_{\theta, r}^{\circ}$. Hence, by Theorem 3.2 , we have

$$
\beta\left(T: \bar{A}_{\theta, p}^{\circ} \times \bar{B}_{\theta, q}^{\circ} \longrightarrow \bar{E}_{\theta, r}^{\circ}\right) \leq 8 \beta\left(T^{\times}:\left(\bar{E}_{\theta, r}^{\circ}\right)^{*} \longrightarrow \mathcal{L}\left(\bar{A}_{\theta, p}^{\circ} \times \bar{B}_{\theta, q}^{\circ}, \mathbb{K}\right)\right)
$$


Let $X_{j}=\mathcal{L}\left(A_{j}^{\circ} \times B_{j}^{\circ}, \mathbb{K}\right), j=0,1$. Since $T: A_{j}^{\circ} \times B_{j}^{\circ} \longrightarrow E_{j}^{\circ}$, is bounded, the restrictions $T^{\times}:\left(E_{j}^{\circ}\right)^{*} \longrightarrow X_{j}$ are also bounded and, by Theorem 3.1, we have

$$
\beta\left(T^{\times}:\left(E_{j}^{\circ}\right)^{*} \longrightarrow X_{j}\right) \leq 4 \beta\left(T: A_{j}^{\circ} \times B_{j}^{\circ} \longrightarrow E_{j}^{\circ}\right), \quad j=0,1 .
$$

Using the formula for the measure of non-compactness of a linear operator interpolated by the real method of Cobos, Fernández-Martínez and Martínez [9, Theorem 1.2] (Banach case) and Fernández-Martínez [14, Theorem 3.1] (quasiBanach case) and (4.2) we derive that

$$
\begin{aligned}
& \beta\left(T^{\times}:\left(\left(E_{0}^{\circ}\right)^{*},\left(E_{1}^{\circ}\right)^{*}\right)_{\theta, \widetilde{r}} \longrightarrow\left(X_{0}, X_{1}\right)_{\theta, \widetilde{r}}\right) \\
& \leq C_{1} \beta\left(T^{\times}:\left(E_{0}^{\circ}\right)^{*} \longrightarrow X_{0}\right)^{1-\theta} \beta\left(T^{\times}:\left(E_{1}^{\circ}\right)^{*} \longrightarrow X_{1}\right)^{\theta} \\
& \quad \leq 4 C_{1} \beta\left(T: A_{0}^{\circ} \times B_{0}^{\circ} \longrightarrow E_{0}^{\circ}\right)^{1-\theta} \beta\left(T: A_{1}^{\circ} \times B_{1}^{\circ} \longrightarrow E_{1}^{\circ}\right)^{\theta} .
\end{aligned}
$$

The duality formulae (2.1), (2.2), (2.3) imply that

$$
\left(\left(E_{0}^{\circ}\right)^{*},\left(E_{1}^{\circ}\right)^{*}\right)_{\theta, \widetilde{r}}=\left(E_{0}^{\circ}, E_{1}^{\circ}\right)_{\theta, r}^{*}
$$

Hence, it follows from Lemma 4.1 and (4.3) that

$$
\begin{aligned}
\beta\left(T^{\times}:\left(E_{0}^{\circ}, E_{1}^{\circ}\right)_{\theta, r}^{*}\right. & \left.\longrightarrow \mathcal{L}\left(\bar{A}_{\theta, p}^{\circ} \times \bar{B}_{\theta, q}^{\circ}, \mathbb{K}\right)\right) \\
\leq & C_{2} \beta\left(T^{\times}:\left(E_{0}^{\circ}, E_{1}^{\circ}\right)_{\theta, r}^{*} \longrightarrow\left(X_{0}, X_{1}\right)_{\theta, \widetilde{r}}\right) \\
& \leq C_{3} \beta\left(T: A_{0}^{\circ} \times B_{0}^{\circ} \longrightarrow E_{0}^{\circ}\right)^{1-\theta} \beta\left(T: A_{1}^{\circ} \times B_{1}^{\circ} \longrightarrow E_{1}^{\circ}\right)^{\theta} .
\end{aligned}
$$

This estimate combined with (4.1) complete the proof.

As a direct consequence of Theorem 4.2 we derive the following compactness result:

Theorem 4.3. Let $\bar{A}=\left(A_{0}, A_{1}\right), \bar{B}=\left(B_{0}, B_{1}\right), \bar{E}=\left(E_{0}, E_{1}\right)$ be Banach couples and let $T \in \mathcal{B}(\bar{A} \times \bar{B}, \bar{E})$ such that $T: A_{j}^{\circ} \times B_{j}^{\circ} \longrightarrow E_{j}^{\circ}$ compactly for $j=0$ or $j=1$. Let $0<\theta<1$ and $0<p, q, r \leq \infty$ such that

$$
\frac{1}{r}= \begin{cases}\frac{1}{p}+\frac{1}{q}-1 & \text { if } \quad p, q \geq 1 \\ \frac{1}{\max (p, q)} & \text { if } \quad p<1 \text { or } q<1 .\end{cases}
$$

Then $T$ may be uniquely extended to a compact bilinear operator from $\left(A_{0}, A_{1}\right)_{\theta, p}^{\circ} \times\left(B_{0}, B_{1}\right)_{\theta, q}^{\circ}$ to $\left(E_{0}, E_{1}\right)_{\theta, r}^{\circ}$.

\section{Acknowledgements}

The authors have been supported in part by by Ministerio de Ciencia e Innovación MTM2017-84058-P (AEI/FEDER, UE).

The authors would like to thank the referee for his/her remarks.

\section{References}

[1] Á. Bényi and R.H. Torres, Compact bilinear operators and commutators, Proc. Amer. Math. Soc. 141 (2013) 3609-3621. 
[2] J. Bergh and J. Löfström, Interpolation Spaces. An Introduction, Springer, Berlin, 1976.

[3] B.F. Besoy and F. Cobos, Interpolation of the measure of non-compactness of bilinear operators among quasi-Banach spaces, J. Approx. Theory 243 (2019) 25-44.

[4] A.P. Calderón, Intermediate spaces and interpolation, the complex method, Studia Math. 24 (1964) 113-190.

[5] B. Carl and I. Stephani, Entropy, Compactness and the Approximation of Operators, Cambridge Univ. Press, Cambridge, 1990.

[6] F. Cobos, L.M. Fernández-Cabrera and A. Martínez, Interpolation of compact bilinear operators among quasi-Banach spaces and applications, Math. Nachr. 291 (2018) 2168-2187.

[7] F. Cobos, L.M. Fernández-Cabrera and A. Martínez, On compactness results of Lions-Peetre type for bilinear operators, Nonlinear Anal. 199 (2020) 111951.

[8] F. Cobos, L.M. Fernández-Cabrera and A. Martínez, A compactness results of Janson type for bilinear operators, J. Math. Anal. Appl. 495 (2021) 124760 .

[9] F. Cobos, P. Fernández-Martínez and A. Martínez, Interpolation of the measure of non-compactness by the real method, Studia Math. 135 (1999) $25-38$.

[10] D.E. Edmunds and W.D. Evans, Spectral Theory and Differential Operators, Clarendon Press, Oxford, 1987.

[11] D.L. Fernandez and E.B. da Silva, Interpolation of bilinear operators and compactness, Nonlinear Anal. 73 (2010) 526-537.

[12] L.M. Fernández-Cabrera and A. Martínez, On interpolation properties of compact bilinear operators, Math. Nachr. 290 (2017) 1663-1677.

[13] L.M. Fernández-Cabrera and A. Martínez, Real interpolation of compact bilinear operators, J. Fourier Anal. Appl. 24 (2018) 1181-1203.

[14] P. Fernández-Martínez, Interpolation of the measure of non-compactness between quasi-Banach spaces, Rev. Mat. Complut. 19 (2006) 477-498.

[15] S. Janson, On interpolation of multilinear operators, in: Function spaces and Applications, in: Springer Lect. Notes in Math., vol. 1302, 1988, pp. 290-302.

[16] G.E. Karadzhov, The interpolation method of "means" for quasinormed spaces, Doklady Acad. Nauk SSSR 209 (1973) 33-36.

[17] H. König, Interpolation of operator ideals with an application to eigenvalue distribution problems, Math. Ann. 233 (1978) 35-48.

[18] J.-L. Lions and J. Peetre, Sur une classe d'espaces d'interpolation, Inst. Hautes Études Sci. Publ. Math. 19 (1964) 5-68. 
[19] M. Mastyło, On interpolation of bilinear operators, J. Funct. Anal. 214 (2004) 260-283.

[20] M. Mastyło, Bilinear interpolation theorems and applications, J. Funct. Anal. 265 (2013) 185-207.

[21] M. Mastyło and E.B. Silva, Interpolation of the measure of non-compactness of bilinear operators, Trans. Amer. Math. Soc. 370 (2018) 8979-8997.

[22] M. Mastyło and E.B. Silva, Interpolation of compact bilinear operators, Bull. Math. Sci. 10 (2020) 2050002.

[23] J. Peetre, Remark on the dual of an interpolation space, Math. Scand. 34 (1974) 124-128.

[24] M.S. Ramanujan and E. Schock, Operator ideals and spaces of bilinear operators, Linear Multilinear Algebra 18 (1985), 307-318.

[25] M.F. Teixeira and D.E. Edmunds, Interpolation theory and measure of noncompactness, Math. Nachr. 104 (1981), 129-135.

[26] R.H. Torres, Q.Y. Xue and J. Yan, Compact bilinear commutators: the quasi-Banach space case, J. Anal. 26 (2018) 227-234.

[27] H. Triebel, Interpolation Theory, Function Spaces, Differential Operators, North-Holland, Amsterdam, 1978. 